\title{
Lumped parameters analysis of coupled kinetics and thermal-hydraulics for small reactors
}

\author{
C. Housiadas* \\ “Demokritos" National Centre for Scientific Research, PO Box 60228, 15310 Agia Paraskevi, Athens, Greece
}

Received 9 July 2001; received in revised form 16 October 2001; accepted 17 October 2001

\begin{abstract}
A useful model is presented that permits to predict with simple means the dynamic response of a small research reactor core. The model uses a lumped parameters approach for the coupled kinetics (point reactor) and thermal-hydraulics, with continuous reactivity feedback due to coolant and fuel temperature effects. The model predictions are qualified by comparing with results from detailed simulations of reactivity insertion transients reported in the literature. Satisfactory results have been obtained for various reactivity insertion rates, up to insertions as high as \$1.5. A simple criterion on the applicability range of the model is suggested, namely, the model can provide reasonably accurate predictions as long as the exit coolant temperature remains below saturation, i.e. no bulk boiling occurs in the core. (C) 2002 Elsevier Science Ltd. All rights reserved.
\end{abstract}

Keywords: Coupled kinetics thermal-hydraulics; Point model; Lumped parameters modelling; Research reactors

\section{Introduction}

The analysis of the transient behaviour of research reactors has received prompt (Woodruff, 1984), as well as recent attention (Mirza et al., 1998; Nasir et al., 1999; Housiadas, 2000) because of its relevance in determining the limits imposed by clad melting temperature. As a rule, the analysis of such transients is carried out with the help of large code systems that simulate the coupled kinetics and thermal-hydraulics of the reactor core. Typical example is the code PARET (Obenchain, 1969; Woodruff, 1984), which is a well-known and widely used code specifically developed for

\footnotetext{
* Tel.: + 30-1-650-3702; fax: + 30-1-653-3431.
}

E-mail address: christos@ipta.demokritos.gr 
predicting the course of transients in research reactors. Also, complicated code systems developed for power reactors have been employed recently in research reactor applications (Woodruff et al., 1997; Hari et al., 2000; Hainoun and Schaffrath, 2001).

Generally, the use of a large code requires a considerable amount of effort and skill with respect, in particular, to input preparation and output processing. This sometimes is hardly affordable by small research reactor operators, who are frequently required to perform transient analyses in order to deal with the safety review of proposed changes. Indeed, contrary to power reactors, research reactor operation is characterized by frequent core modifications, as required to respond to the changing experimental needs. In practice, a modification procedure includes a number of iterations in order to clarify, amend, or supplement the intended change, which obviously increases further the overall computational obligations. Hence, it is particularly desirable for the operator to dispose means to perform simple and realistic transient estimations, even if only for scoping purposes, without the need of resorting every time to a full simulation with a code. The objective of the present work is precisely to provide a simple and accurate enough model for predicting the dynamic response of a small reactor core. The approach is based on a lumped parameters modelling of both the kinetic and thermal-hydraulic effects.

Most models for coupled kinetics and thermal-hydraulics consider a point reactor, and hence, the kinetic effects are described, anyway, with a lumped parameters approach. Instead, thermal-hydraulics are computed on the basis of a one-, or multidimensional core description. Lumped parameters models for thermal-hydraulics are generally considered only for qualitative studies of reactor dynamics. Relevant examples can be found in standard texts (Duderstadt and Hamilton, 1976), and include the Newton's law of cooling model, the adiabatic model, and the constant power removal model. In these simplified models temperature effects are accounted for through a single effective temperature for the whole core. In the present analysis, temperature effects are described by considering two distinct temperature regions, corresponding respectively to the coolant and the fuel. This enables reactivity feedback to include all the major contributions, namely, moderator temperature/density effects and fuel temperature effects. In line with the point model concept, coolant temperature and fuel temperature are both assumed to be functions separable in space and time. The space dependence is postulated to be the static distribution corresponding to a one-dimensional (in the axial direction) core. As shown below, the model is computationally economic, can be implemented with little numerical and development effort, and provides predictions with an accuracy sufficient for practical purposes. The model can accommodate the usual transient initiators, e.g. loss of flow or reactivity insertion. The present work considers only the severe case of unprotected reactivity insertion transients. However, as it will become apparent, the model can be easily adapted to analyse also other types of transient. Note that in reactivity transients the inserted reactivity needs to be pre-specified in the model, hence, in analysing practical situations some neutronic calculations will be required first. Thus, the proposed model does not circumvent the need of using neutronic codes, but alleviates substantially the next step, namely the calculation of the coupled kinetic and thermal-hydraulic response of the core. 
The paper is organized in the following way. Section 2 describes the modelling approach. Section 3 illustrates the model capabilities by comparing with detailed results on reactivity insertion transients taken from the literature. Finally, the conclusions are summarized in Section 4.

\section{Modelling}

Consider a typical pool-type research reactor with MTR-type fuel elements of rectangular geometry, cooled and moderated with light water. A one-dimensional core is considered, consisting of a cooling channel of width $2 b$ and a fuel plate of width $2 d$. Incompressible slug flow of velocity $U$ is assumed to take place in the channel, whereas convective heat transfer is assumed to take place on the plate surface through a constant heat transfer coefficient $h$. The equations for the local coolant temperature $\hat{T}_{c}$ and fuel temperature $\hat{T}_{f}$ are

$$
\begin{aligned}
& \rho_{c} c_{c} \frac{\partial \hat{T}_{c}}{\partial t}+\rho_{c} c_{c} U \frac{\partial \hat{T}_{c}}{\partial z}=\frac{h}{b}\left(\hat{T}_{f}-\hat{T}_{c}\right) \\
& \rho_{f} c_{f} \frac{\mathrm{d} \hat{T}_{f}}{\mathrm{~d} t}=-\frac{h}{d}\left(\hat{T}_{f}-\hat{T}_{c}\right)+\hat{P}
\end{aligned}
$$

where $t$ is time, $z$ the axial coordinate, $\rho$ the density, $c$ the specific heat, and the subscripts $c$ and $f$ denote respectively the coolant and the fuel. In Eq. (2) $\hat{P}$ is the local power density (power per unit fuel volume), which is assumed to have the profile of the fundamental eigenfunction (cosine form). The mean (core-averaged) value of an axially dependent quantity like $\hat{T}_{c}$ is given by

$$
T_{c}(t)=\frac{1}{H} \int_{0}^{H} \hat{T}_{c}(z, t) d z .
$$

By applying the above rule on both sides of Eqs. (1) and (2), one obtains

$$
\begin{aligned}
& \rho_{c} c_{c} \frac{\mathrm{d} T_{c}}{\mathrm{~d} t}+\rho_{c} c_{c} U \frac{1}{H}\left(T_{\text {out }}-T_{p}\right)=\frac{h}{b}\left(T_{f}-T_{c}\right) \\
& \rho_{f} c_{f} \frac{\mathrm{d} T_{f}}{\mathrm{~d} t}=-\frac{h}{d}\left(T_{f}-T_{c}\right)+P
\end{aligned}
$$

where $T_{c}$ is the mean coolant temperature, $T_{\text {out }}$ the outlet coolant temperature, $T_{\text {out }}=\hat{T}_{c}(z=H, t), T_{p}$ the inlet coolant temperature (equivalent to pool temperature), $T_{p}=\hat{T}_{c}(z=0, t), T_{f}$ the mean fuel temperature, and $P$ the mean reactor power. In agreement with the point kinetics model, assume that the shape of functions $\hat{T}_{c}(z, t)$ and $\hat{T}_{f}(z, t)$ remains unchanged with time, and identical to the profile corresponding to static conditions. Considering the steady-state solutions of Eqs. (1) and (2) it can be shown that the axial profiles can be expressed as follows 


$$
\begin{aligned}
& \hat{T}_{c}=T_{p}+\left(T_{c}-T_{p}\right)\left[1-\cos \left(\frac{\pi z}{H}\right)\right] \\
& \hat{T}_{f}=T_{p}+\left(T_{c}-T_{p}\right)\left[1-\cos \left(\frac{\pi z}{H}\right)\right]+\frac{\pi}{2}\left(T_{f}-T_{c}\right) \sin \left(\frac{\pi z}{H}\right)
\end{aligned}
$$

where $H$ is core height. The above expressions permit to approximate the axial temperature distributions of coolant and fuel element with the help of the mean temperatures $T_{c}$ and $T_{f}$, i.e. the lumped parameters. More specifically, Eq. (6) permits to write

$$
T_{c}=\frac{T_{\mathrm{out}}+T_{p}}{2}
$$

which enables Eq. (1) to be expressed in terms of the lumped parameters $T_{c}$ and $T_{f}$ as follows

$$
\rho_{c} c_{c} \frac{\mathrm{d} T_{c}}{\mathrm{~d} t}+2 \rho_{c} c_{c} \frac{U}{H}\left(T_{c}-T_{p}\right)=\frac{h}{b}\left(T_{f}-T_{c}\right)
$$

On the other hand, Eq. (7) can be used to determine the maximum fuel temperature point. By differentiating Eq. (7) with respect to $z$, equating to zero, and solving for $z$, it follows that the axial location at which the maximum fuel temperature occurs is

$$
z_{m}=\frac{H}{2}+\frac{H}{\pi} \tan ^{-1} \frac{2}{\pi} \frac{T_{c}-T_{p}}{T_{f}-T_{c}}=\frac{H}{2}+\frac{H}{\pi} \tan ^{-1} \frac{h H}{\pi \rho_{c} c_{c} U b d} .
$$

In the present simulations the heat transfer coefficient $h$ is determined from the Dittus-Boelter correlation for turbulent-flow convection. The core inlet temperature (or pool temperature) $T_{p}$ is normally a constant specified as an input parameter. However, the option of pool heating has been also accommodated to analyse conditions in which pool temperature rises because of simultaneous loss of secondary cooling. This can be accomplished by introducing an additional differential equation, based on a simple heat balance over the pool volume

$$
\rho_{c} c_{c} V_{p} \frac{\mathrm{d} T_{p}}{\mathrm{~d} t}=V_{f} P
$$

where $V_{p}$ is the volume of the pool and $V_{f}$ is the fuel volume.

The reactor power is calculated from a point reactor kinetics model with one group of delayed neutrons

$$
\frac{\mathrm{d} P}{\mathrm{~d} t}=\frac{\rho(t)-\beta}{\Lambda} P+\lambda C
$$




$$
\frac{\mathrm{d} C}{\mathrm{~d} t}=\frac{\beta}{\Lambda} P-\lambda C
$$

where $\rho(t)$ is the reactivity, $\lambda$ the precursor decay constant, $\beta$ the delayed neutron fraction, $\Lambda$ the mean neutron generation time, and $C$ is the precursors number density (times a constant). Reactivity $\rho(t)$ is determined by calculating continuous reactor feedback as follows

$$
\rho(t)=\rho_{e x}(t)+a_{M} \delta \rho_{c}-a_{T} \delta T_{c}-a_{F} \delta T_{f}
$$

In the above expression, the first term in the right-hand side is the externally introduced reactivity and the remaining terms are the various feedback contributions. Feedback effects are induced by changes of coolant density $\delta \rho_{c}$ (due to temperature changes), changes of coolant temperature $\delta T_{c}$ (spectrum effects only), and changes of fuel temperature $\delta T_{f}$ (Doppler effects). The corresponding coefficients of reactivity are respectively $a_{M}, a_{T}$, and $a_{F}$. Eq. (14) implies that there are only small reactivity changes that can be expressed linearly with the help of constant coefficients of reactivity. Strictly speaking, such approach is not applicable to transient cases. However, neutronic calculations performed for the Greek Research Reactor-1 (GRR-1, see Table 1 below) have shown that the slopes $\partial \rho / \partial T_{c}, \partial \rho / \partial T_{f}$, and $\partial \rho / \partial \rho_{c}$ remain practically constant over extended ranges of variation for parameters $T_{c}, T_{f}$, and $\rho_{c}$, respectively. Therefore, Eq. (14) remains to a very good approximation valid also for large changes and this justifies its use in transient analyses. In applying Eq. (14) the changes have to be determined by considering the variations of the mean coolant and fuel temperatures, namely $\delta \rho_{c}=\rho_{c}\left(T_{c}\right)-\rho_{c}\left(T_{c}(t=0)\right), \quad \delta T_{c}=$ $T_{c}-T_{c}(t=0)$, and $\delta T_{f}=T_{f}-T_{f}(t=0)$. The approach of using mean (whole volume) quantities to determine reactivity feedback, besides being consistent with the

Table 1

Input data used in the lumped parameters model calculations, as compiled from the literature (all cases are concerned with pool-type research reactors cores with MTR-type fuel elements)

\begin{tabular}{llll}
\hline & Mirza et al. (1998) & Nasir et al. (1999) & Housiadas (2000) \\
\hline Reactor & "Typical MTR" & PARR-1 & GRR-1 \\
Fuel & LEU & LEU & LEU \\
Inlet coolant temperature $\left({ }^{\circ} \mathrm{C}\right)$ & 32 & 38 & 38, or 44 \\
Inlet coolant pressure $($ bar) & 1.703 & 1.703 & 1.796 \\
Coolant mass velocity $\left(\mathrm{kg} / \mathrm{m}^{2} \mathrm{~s}\right)$ & 2880 & 2868 & 1167 \\
$H(\mathrm{~mm})$ & 625 & 625 & 625.5 \\
$2 b, 2 d(\mathrm{~mm})$ & $2.10,1.50$ & $2.10,1.27$ & $2.90,1.52$ \\
Fuel volume $\left(\mathrm{m}^{3}\right)$ & $3.862 \times 10^{-2}$ & $3.574 \times 10^{-2}$ & $3.508 \times 10^{-2}$ \\
$\Lambda(\mu \mathrm{s})$ & 55.96 & 42.0 & 57.6 \\
$\beta$ & $7.2754 \times 10^{-3}$ & $7.275 \times 10^{-3}$ & $6.60 \times 10^{-3}$ \\
$a_{M}(\$ / \mathrm{g} \mathrm{cm}$ & $-3)$ & 52.90 & 35.799 \\
$a_{T}\left(\$ /{ }^{\circ} \mathrm{C}\right)$ & 38.142 & $8.2000 \times 10^{-3}$ & $1.341 \times 10^{-2}$ \\
$a_{F}\left(\$ /{ }^{\circ} \mathrm{C}\right)$ & $8.2186 \times 10^{-3}$ & $2.625 \times 10^{-4}$ & $2.370 \times 10^{-3}$ \\
Pool volume $\left(\mathrm{m}^{3}\right)$ & $3.180 \times 10^{-3}$ & - & 300 \\
\hline
\end{tabular}


lumped parameters modelling employed here, is also consistent with recent theoretical developments on the estimation of the moderator temperature coefficient (Antonopoulos-Domis and Housiadas, 1999; Housiadas and Antonopoulos-Domis, 1999).

Eqs. (5), (9), (12-14) define completely the model sought, in terms of lumped parameters. In case that pool heating is enabled Eq. (11) must be also included. The above system, following specification of initial values for $T_{c}, T_{f}, P, C$, (and eventually $T_{p}$ ) is solved numerically to provide the reactor transient response to an externally specified reactivity forcing $\rho_{e x}(t)$. The numerical integration can be accomplished in a straightforward manner by using a forth-order Runge-Kutta method and treating explicitly the right members of the equations. Another option, which is more convenient, is to resort to the extensive capabilities provided today by commercial software packages for modelling dynamical and control systems, such as Matlab's Simulink. In fact, we have used Simulink to generate our results, which permitted to implement the numerics of the model with minimal effort.

The numerics of the model were verified by comparing with known analytical solutions. The kinetics part of the model was tested considering the well-known analytical solution for a reactivity jump without feedback. The thermal-hydraulics part was qualified with the help of available analytical results on transient slug flow convection between parallel plates (Siegel, 1959). In both comparisons, the numerically generated results agreed almost exactly with the analytical solutions.

\section{Results}

To assess the capabilities of the model presented above, we reproduced relevant transient cases that were analysed recently in the literature (Mirza et al., 1998; Nasir et al., 1999). In these works, unprotected (without scram) reactivity insertion transients in typical pool-type research reactors were analysed with the help of the code PARET. As mentioned in the Introduction, code PARET is a major coupled kinetics and thermal-hydraulics code for predicting the course of non-destructive transients in research reactors (Woodruff, 1984).

Fig. 1 shows the model predictions for reactivity (a), power (b), outlet coolant temperature $T_{\text {out }}$ and maximum fuel temperature $T_{\text {fmax }}$ (c), following a step reactivity insertion of $\$ 1.5$ without scram. The temperatures $T_{\text {out }}$ and $T_{\text {fmax }}$ have been determined from the primary model variables $T_{c}$ and $T_{f}$ with the help of Eqs. (6), (7) and (10). For comparison, the numerical results of Mirza et al. (1998) are also plotted. The calculations have been performed using the reactor and kinetic parameters as reported in the paper of Mirza et al. (1998). The input data used are summarized in Table 1. Inspection of Fig. 1 suggests that the lumped parameters model reproduces fairly closely the code predictions. In particular, the times corresponding to the power and temperature peaks are determined in satisfactory agreement with the code, as well as the prediction of the maximum fuel temperature. The latter is the most important parameter in the safety assessments. The reactivity deep (by action of feedback) is underestimated by the model, which is accompanied by a correspondingly marked overestimation of the peak power. The conservatism of the 

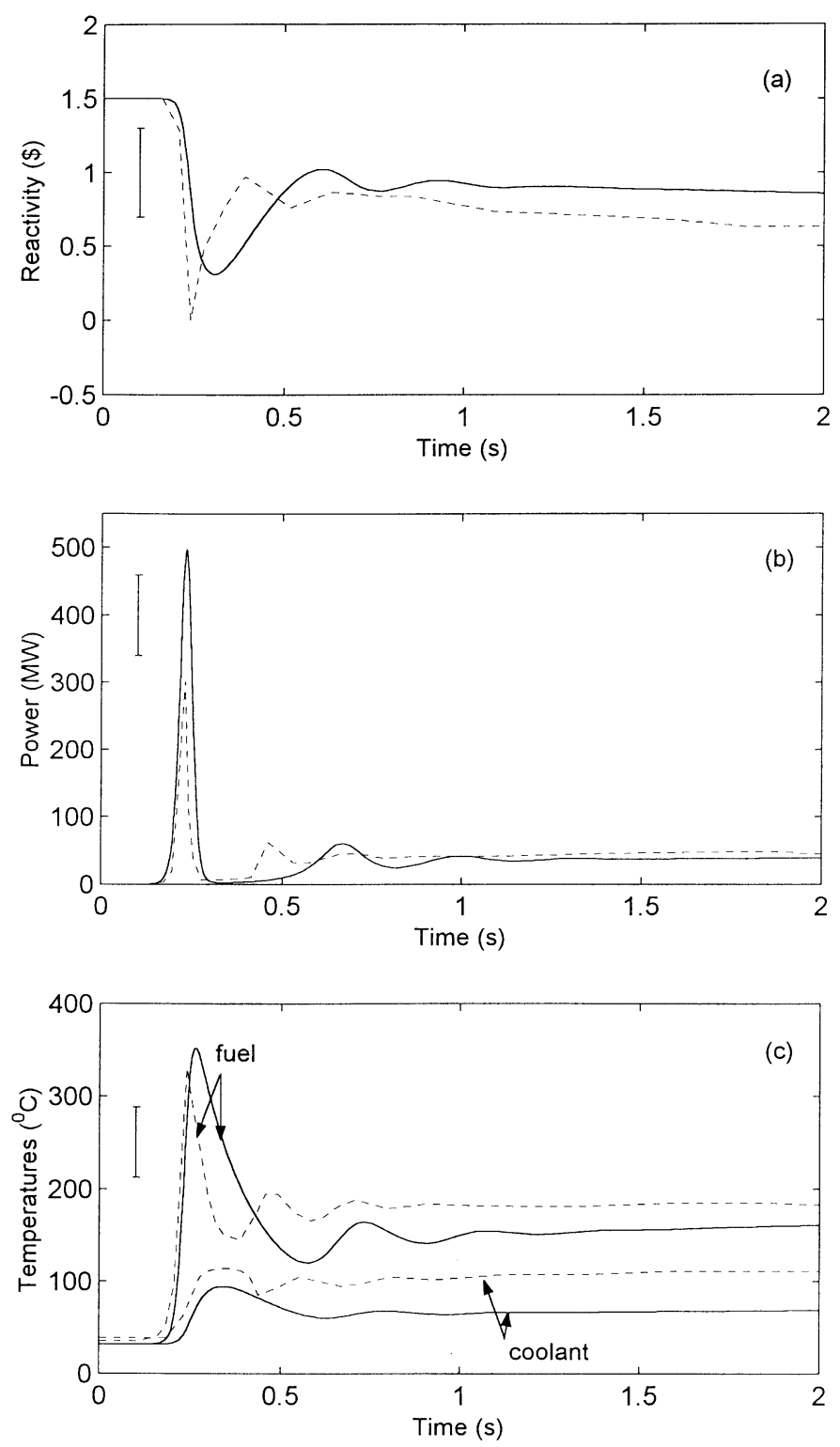

Fig. 1. Step reactivity insertion of $\$ 1.5$ with scram disabled (initial power $=1 \mathrm{~W}$ ). Responses of reactivity (a), power (b), coolant and fuel temperatures (c), as predicted with the present lumped parameters model (solid lines) and comparison with the detailed simulation results of Mirza et al. (1998) (dashed lines).

model is attributed to the use of purely single phase thermal-hydraulics, which obviously ignore void feedback effects. Note that in the considered transient some voiding due to subcooled boiling is taking place, because fuel temperature exceeds largely the onset of nucleate boiling (ONB) point. Also, discrepancies are apparent 
in the response of coolant and fuel temperatures in the decay phase of the transient. However, the observed discrepancies cannot be considered as unacceptably large. This can be attested by inspecting the error bars shown in Fig. 1, which represent the uncertainty of the code predictions. The latter was inferred from an extensive comparison campaign between PARET calculations and experimental results and was determined to be of about 30\% (Obenchain, 1969). Hence, the lumped parameters model proved reasonably accurate in determining the course and consequences of the considered severe reactivity transient. Note also that the considered transient is the most unfavourable case to analyse because, as shown next, corresponds to the applicability limit of the model.

To determine the validity range of the model we compared the model predictions with the sensitivity analysis results of Nasir et al. (1999). The latter used the code PARET to investigate the response of a research reactor to various ramp reactivity insertion conditions. The comparison is provided in Table 2 in terms of peak power and maximum coolant and fuel temperatures attained, together with the times to reach peak conditions. As before, the required input data were taken from the paper of Nasir et al. (1999) (see Table 1). Since the paper does not document results for ramp reactivity insertions less than $\$ 1.5 / 0.5 \mathrm{~s}$, we performed some supplementary PARET simulations (cases of Table 2 marked with an asterisk). The GRR-1 is considered as the reference design in these supplementary simulations (input data also given in Table 1). Full details on the reactor description can be found in Housiadas (2000). The results of Table 2 indicate that the lumped parameters model

Table 2

Comparison between the predictions obtained with the proposed lumped parameters model and the code generated results (in parentheses) of Nasir et al. (1999) for various ramp reactivity insertion rates (scram disabled, initial power $1 \mathrm{~W}$ )

\begin{tabular}{|c|c|c|c|c|c|c|}
\hline \multirow[t]{2}{*}{ Reactivity $\$ / 0.5 \mathrm{~s}$} & \multicolumn{2}{|l|}{ Power } & \multicolumn{2}{|c|}{ Coolant temperature ${ }^{a}$} & \multicolumn{2}{|c|}{ Fuel temperature } \\
\hline & Peak (MW) & Time (s) & $\operatorname{Max}\left({ }^{\circ} \mathrm{C}\right)$ & Time (s) & $\operatorname{Max}\left({ }^{\circ} \mathrm{C}\right)$ & Time (s) \\
\hline $1.1^{*}$ & $\begin{array}{c}16.67 \\
(21.82)\end{array}$ & $\begin{array}{c}1.703 \\
(1.498)\end{array}$ & $\begin{array}{c}50.1 \\
(59.4)\end{array}$ & $\begin{array}{c}2.071 \\
(2.802)\end{array}$ & $\begin{array}{c}93.3 \\
(98.9)\end{array}$ & $\begin{array}{c}1.872 \\
(1.698)\end{array}$ \\
\hline $1.2^{*}$ & $\begin{array}{c}63.07 \\
(72.50)\end{array}$ & $\begin{array}{c}1.157 \\
(1.120)\end{array}$ & $\begin{array}{c}62.9 \\
(71.8)\end{array}$ & $\begin{array}{c}1.431 \\
(1.479)\end{array}$ & $\begin{array}{c}171.5 \\
(146.9)\end{array}$ & $\begin{array}{c}1.252 \\
(1.189)\end{array}$ \\
\hline 1.5 & $\begin{array}{c}457.8 \\
(404.2)\end{array}$ & $\begin{array}{c}0.626 \\
(0.611)\end{array}$ & $\begin{array}{c}99.9 \\
(115.2)\end{array}$ & $\begin{array}{c}0.723 \\
(0.738)\end{array}$ & $\begin{array}{c}358.4 \\
(290.5)\end{array}$ & $\begin{array}{c}0.653 \\
(0.618)\end{array}$ \\
\hline 1.6 & $\begin{array}{c}673.9 \\
(525.9)\end{array}$ & $\begin{array}{c}0.584 \\
(0.571)\end{array}$ & $\begin{array}{c}118.5 \\
(115.2)\end{array}$ & $\begin{array}{c}0.683 \\
(0.677)\end{array}$ & $\begin{array}{c}447.9 \\
(323.8)\end{array}$ & $\begin{array}{c}0.609 \\
(0.575)\end{array}$ \\
\hline 1.7 & $\begin{array}{c}938.3 \\
(651.9)\end{array}$ & $\begin{array}{c}0.553 \\
(0.541)\end{array}$ & $\begin{array}{c}129.6 \\
(115.2)\end{array}$ & $\begin{array}{c}0.646 \\
(0.638)\end{array}$ & $\begin{array}{c}541.5 \\
(347.6)\end{array}$ & $\begin{array}{c}0.575 \\
(0.543)\end{array}$ \\
\hline 1.8 & $\begin{array}{l}1241.2 \\
(775.1)\end{array}$ & $\begin{array}{c}0.528 \\
(0.516)\end{array}$ & $\begin{array}{c}144.7 \\
(115.2)\end{array}$ & $\begin{array}{c}0.619 \\
(0.584)\end{array}$ & $\begin{array}{c}636.9 \\
(368.0)\end{array}$ & $\begin{array}{c}0.547 \\
(0.518)\end{array}$ \\
\hline 1.9 & $\begin{array}{l}1540.7 \\
(838.1)\end{array}$ & $\begin{array}{c}0.507 \\
(0.496)\end{array}$ & $\begin{array}{c}158.4 \\
(115.2)\end{array}$ & $\begin{array}{c}0.608 \\
(0.569)\end{array}$ & $\begin{array}{c}724.5 \\
(377.2)\end{array}$ & $\begin{array}{c}0.524 \\
(0.497)\end{array}$ \\
\hline
\end{tabular}

*Simulation results obtained with code PARET considering GRR-1 research reactor.

a Saturation temperature at 1.7 bar $=115.2^{\circ} \mathrm{C}$. 
provides quite accurate predictions for the peak points of power and core temperature for reactivity insertions up $\$ 1.5$. At larger reactivity insertions the model performances deteriorate rapidly, and unrealistic results are provided. This is connected with the occurrence of significant void (vapour) generation in the core, as result of
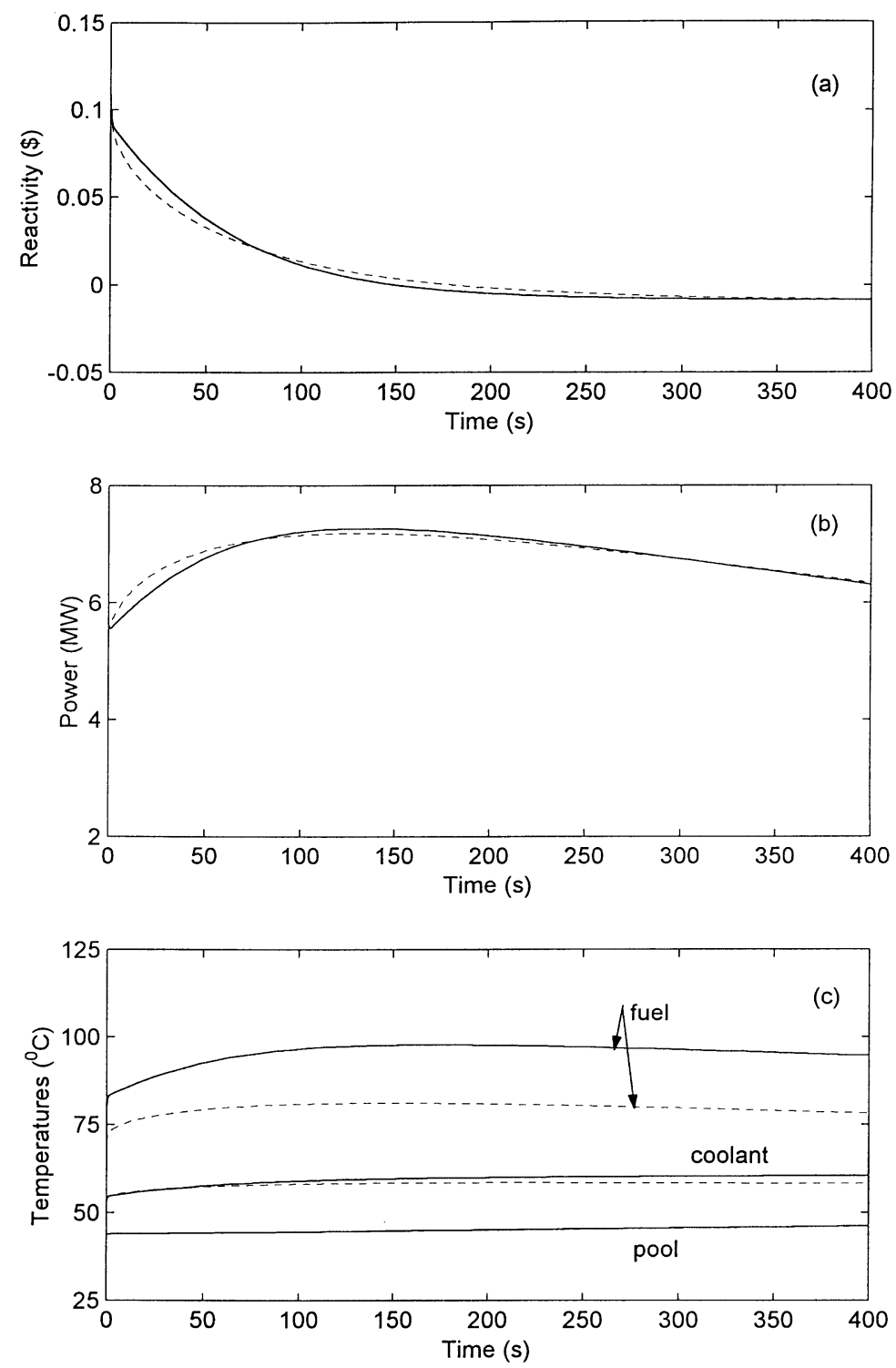

Fig. 2. Step reactivity insertion of $\$ 0.11$ without scram and simultaneous failure of secondary cooling (initial power $=5 \mathrm{MW}$ ). Responses of reactivity (a), power (b), coolant, fuel and pool temperatures (c), as predicted with the present lumped parameters model (solid lines) and comparison with the simulation results generated with code PARET (dashed lines) (Stamatelatos and Housiadas, 1999). 
the significant energy release that accompanies such large reactivity insertions. Indeed, inspection of Table 2 indicates that coolant temperature, as predicted from the detailed code simulations, reaches saturation for $\rho_{e x} \geq \$ 1.5$, whereas for $\rho_{e x}<$ $\$ 1.5$ remains highly subcooled. For $T_{\text {out }}<T_{\text {sat }}$ ( $T_{\text {sat }}$ is saturation temperature) limited voiding is produced in the core due to subcooled boiling. Instead, when $T_{\text {out }} \geq$ $T_{\text {sat }}$ bulk boiling is taking place and significant void is generated in the core. Clearly, the latter conditions cannot be simulated by the present model because two-phase flow effects are completely ignored in the simple thermal-hydraulics description used. On that basis, a simple criterion on the applicability range of the model can be established, namely, the model remains valid as long as the predicted exit coolant temperature does not exceed the saturation temperature, i.e. $T_{\text {out }}<T_{\text {sat }}$.

The advantages of the proposed modelling approach become apparent in the case of long transients of moderate severity. Such analyses are frequently required in research reactor operation. As example consider the case of flooding of an experimental air channel with water, with simultaneous loss of secondary cooling. In fact, this case has arisen in the safety review of an in-core irradiation facility in GRR-1 reactor. Obviously, the whole analysis required the use of a transport code prior to using the proposed model, to estimate the reactivity insertion worth. Code MCNP was used and the inserted reactivity was estimated to be $\$ 0.11$ (Stamatelatos and Housiadas, 1999). The reactor is initially operating at the nominal power level of $5 \mathrm{MW}$ and the trip on over-power is assumed to fail. The results obtained with the present model are compared to those obtained with code PARET in Fig. 2. In this case pool heating is enabled and, therefore, Eq. (11) is included in the modelling. As can be seen, the proposed model reproduces very closely the code results in terms of power, reactivity, or coolant temperature. There is some disagreement in terms of fuel temperature, which, nevertheless is beyond any practical concern because the differences are of the order of $10{ }^{\circ} \mathrm{C}$. Note also that reactivity becomes negative late in the transient although reactor trip has failed. Since secondary cooling is not available pool temperature increases gently, but constantly, as indeed can be observed in Fig. 2c. This temperature rise induces negative reactivity causing a power decrease in later times as can be clearly seen in the results of Fig. 2 b.

\section{Conclusions}

A useful model is presented that permits to predict with simple means the behaviour of a small research reactor core during transient conditions. The model is based on a lumped parameters description of both the kinetics (point model) and thermal-hydraulics. The model is completely defined by Eqs. (5), (9), (12)-(14), supplemented eventually with Eq. (11) if pool heating is enabled. The above initial value problem gives rise to a dynamical system that can be integrated numerically easily and with little development effort.

In the present work the model was applied to the analysis of unprotected transients induced by an insertion of reactivity. However, the model can be easily adapted to analyse also other types of transients. 
Based on comparisons between model predictions and detailed simulations of various reactivity insertion transients analysed in the literature, the following conclusions can be drawn. The proposed model provides a reasonably accurate means for determining the transient behaviour of a research reactor, provided that no significant vapour generation takes place in the core. The model was demonstrated to be applicable for various reactivity insertion rates, up to insertions as high as $\$ 1.5$. A simple criterion on the applicability range of the model is suggested, namely, the model can be used to provide reasonably accurate predictions as long as $T_{\text {out }}<T_{\text {sat }}$, i.e. no bulk boiling occurs in the core. The recommended applicability range covers most of the transient analysis requirements encountered in practice, excluding only those transients associated with extreme conditions.

\section{Acknowledgements}

I would like to thank Professor Antonopoulos-Domis for his support.

\section{References}

Antonopoulos-Domis, M., Housiadas, C., 1999. Moderator temperature coefficient of reactivity in Pressurized Water Reactors: theoretical investigation and numerical simulations. Nuclear Science and Engineering 132, 337-345.

Duderstadt, J.J., Hamilton, L.J., 1976. Nuclear Reactor Analysis. John Wiley \& Sons, New York.

Hari, S., Hassan, Y.A., Tu, J., 2000. Analysis of transient events without scram in a research reactor using the RELAP/MOD3.2 computer code. Nuclear Technology 130, 296-309.

Hainoun, A., Schaffrath, A., 2001. Simulation of subcooled flow instability for high flux research reactors using the extended code ATHLET. Nuclear Engineering and Design 207, 163-180.

Housiadas, C., 2000. Simulation of loss-of-flow transients in research reactors. Annals of Nuclear Energy 27, 1683-1693.

Housiadas, C., Antonopoulos-Domis, M., 1999. The effect of fuel temperature on the estimation of the moderator temperature coefficient in PWRs. Annals of Nuclear Energy 26, 1395-1405.

Mirza, A.M., Khanam, S., Mirza, N.M., 1998. Simulation of reactivity transients in current MTRs. Annals of Nuclear Energy 25, 1465-1484.

Nasir, R., Mirza, N.M., Mirza, S.M., 1999. Sensitivity of reactivity insertion limits with respect to safety parameters in a typical MTR. Annals of Nuclear Energy 26, 1517-1535.

Obenchain, C.F., 1969. PARET-A program for the analysis of reactor transients. Idaho National Engineering Laboratory, Report IDO-17282.

Siegel, R., 1959. Transient heat transfer for laminar slug flow in ducts. Transactions ASME-Journal of Applied Mechanics 26, 140-142.

Stamatelatos, I.E., Housiadas, C., 1999. Reactivity effects due to GRR-1 "Demokritos" reactor core modifications for in-core sample irradiation experiments. NCSR “Demokritos”, Report DEMO 6/99.

Woodruff, W.L., 1984. A kinetics and thermal-hydraulics capability for the analysis of research reactors. Nuclear Technology 64, 196-206.

Woodruff, W.L., Hanan, N.A., Smith, R.S., Matos, J.E., 1997. A comparison of the PARET/ANL and RELAP/MOD3 codes for the analysis if IAEA benchmark transients and the SPERT experiments. Transactions of the American Nuclear Society 77, 92-93. 